\title{
A study of Kapteyn's star
}

\author{
E. Kotoneva ${ }^{1,2}$, K. Innanen ${ }^{2}$, P. C. Dawson ${ }^{3,4}$, P. R. Wood ${ }^{5}$, and M. M. De Robertis ${ }^{2}$ \\ 1 Tuorla Observatory, Väisäläntie 20, 21500 Piikkiö, Finland \\ e-mail: eira.kotoneva@tyks.fi \\ 2 Department of Physics and Astronomy, York University, 4700 Keele Street, Toronto, ON M3J 1P3, Canada \\ 3 Department of Physics, Trent University, Peterborough, ON, K9J 7B8, Canada \\ ${ }^{4}$ Guest user, Canadian Astronomy Data Centre, operated by the Herzberg Institute of Astrophysics, \\ National Research Council of Canada \\ ${ }^{5}$ Research School of Astronomy \& Astrophysics, Mount Stromlo Observatory, Cotter Road, Weston ACT 2611, Australia
}

Received 1 November 2004 / Accepted 15 February 2005

\begin{abstract}
We present a review of the current knowledge of Kapteyn's Star (KS) - a nearby, low-metallicity M-dwarf, with an eccentric and retrograde Galactic orbit. A brief survey of its spectroscopic properties is provided, together with an analysis of its Galactic orbit in a Galaxy model that incorporates resonances. We propose that KS may have once belonged to a dwarf spheroidal galaxy that merged with the Galaxy, and whose present remnant, if it still exists, is a globular cluster similar to $\omega$ Cen.
\end{abstract}

Key words. stars: late-type - stars: general

\section{Introduction}

Kapteyn's Star (hereafter, KS) is among the most interesting of the Sun's nearest neighbours. Superficially, it appears to be a fairly unexceptional M1 V star, and it was classified as such, on the basis of its TiO band strengths, by Adams \& Joy (1924), who compared it to Groombridge 34 (LHS 3, GJ 15A). Kuiper (1940) included KS in his list of the first three M-subdwarfs to be definitely identified (the others were Ross 578 = LHS 20, and Wolf $1106 \equiv$ LHS 64), and commented that all three stars appeared to be subluminous by at least $2 \mathrm{mag}$.

Its proper motion is very large, second only to that of Barnard's Star. Combined with the star's large radial velocity and (precise) parallax, it yields a space motion that is truly remarkable; KS exhibits one of the relatively few wellestablished retrograde Galactic orbits, at least among the stars that populate the immediate solar neighbourhood. A recent comprehensive study of the spectrum of KS by Woolf $\&$ Wallerstein (2004) yielded a weighted mean metallicity $[\mathrm{M} / \mathrm{H}]=-0.98 \pm 0.10$, subsequently revised to -0.86 . That, when viewed in the context of a Galactic orbit that is near the chaotic threshold, makes it possible to address the question of the star's origin and history. This paper is organized as follows: Sect. 2 is a review of some of the literature pertaining to KS, concluding with our adopted physical parameters. In Sect. 3, the optical and near-IR spectra are discussed, and compared with theoretical models. The Galaxy model used to compute the orbit of KS is described in Sect. 4, and in Sect. 5, possible Galactic orbits are investigated. Section 6 presents a discussion and conclusions.

\section{Kapteyn's star}

Kapteyn's Star (GJ 191, LHS 29) is a nearby M-dwarf whose high proper motion (until 1916, the largest known) was discovered by J. C. Kapteyn (1897) as a byproduct of his work on the Cape Photographic Durchmusterung. Since then, it has attracted considerable attention, partly because its high space velocity (first reported by Curtis 1909) relative to the Sun has been taken as an indication of halo membership; it is probably the nearest known halo object. Wing et al. (1976) carried out a study of its spectrum, commenting on the strength of the $\mathrm{CaH}$ and $\mathrm{MgH}$ bands, and the weakness of the TiO bands; they estimated its effective temperature to be $3800 \mathrm{~K}$. While they were unable to arrive at a definite value for the star's metallicity, they suggested that Kapteyn's Star might be metal-poor, and that the spectral type might not be a reliable temperature indicator. Mould (1976) carried out the first spectroscopic abundance analysis of KS. He estimated $[\mathrm{Fe} / \mathrm{H}]=-0.50 \pm 0.30$, and $[\mathrm{Ti} / \mathrm{H}]=-0.20 \pm 0.30$ from the neutral metal lines; $[\mathrm{M} / \mathrm{H}]=-0.50 \pm 0.30$ from the $\mathrm{TiO}$ bands; and $[\mathrm{Ca} / \mathrm{H}]=$ $-1.20 \pm 0.50$ from the $\mathrm{Ca}$ II infrared triplet. Then, assuming scaled solar abundance patterns, he arrived at a weighted mean $[\mathrm{M} / \mathrm{H}]=-0.55 \pm 0.15$ (p. e.), with $\theta_{\mathrm{eff}}=5040 \mathrm{~K} / T_{\mathrm{eff}}=1.43$. Proust \& Foy (1988) analyzed two supposed members of the Kapteyn's Star moving group, HD 44007 and HD 103036, and derived $[\mathrm{Fe} / \mathrm{H}]=-0.65$ and $[\mathrm{Fe} / \mathrm{H}]=-1.20$, respectively. The difference between these values, considered in the light of Eggen's (1977) own estimate of the group's metal abundance, namely, $[\mathrm{Fe} / \mathrm{H}]=-1.80$, led them to doubt the reality of the group. Eggen (1978) drew attention to a similarity, evident in 
the $M_{V}, R-I$ and $M_{I}, R-I$ planes, between members of the KS moving group and members of the globular cluster $\omega$ Cen this will be of interest in the context of the discussion in Sect. 6. Gizis (1997) classified KS as sdM1.0, and estimated [M/H] = -1.50 , together with $T_{\text {eff }}=3700 \mathrm{~K}$. Krawchuk et al. (2000) attempted to arrive at a temperature and metallicity by matching the observed colours of KS with those computed from synthetic spectra based on the NextGen models (Hauschildt et al. 1999). They found $T_{\text {eff }}$ near $3500 \mathrm{~K}$, and $[\mathrm{M} / \mathrm{H}]$ close to -1.80 , depending on the choice of the colour-colour diagram. This was consistent with Eggen's (1997) revision of the mean metallicity of the KS moving group to $P[\mathrm{Fe} / \mathrm{H}]=-1.65 \pm 0.12$ (s.e.). Jones et al. (2002) observed the spectrum of KS and three other $\mathrm{dM}$ stars between 2.5 and $3 \mu \mathrm{m}$, using the ISO shortwavelength spectrometer. The observed spectral energy distributions (SEDs) were then compared with synthetic spectra based on data from the Partridge \& Schwenke (1997) line lists for water vapour. In the case of KS, they found the best match between observed and synthetic spectra for $T_{\text {eff }}=3700 \pm 100 \mathrm{~K}$ and $[\mathrm{Fe} / \mathrm{H}]=-1.00 \pm 0.30$. They speculated that the relatively high temperature deduced for KS (and for the other $\mathrm{dM}$ stars in their sample) might be the result of problems in the Partridge and Schwenke line lists - problems resulting in overestimates of water vapour band strengths at any given temperature. More recently, the spectrum of KS has been analyzed by Woolf \& Wallerstein (2004), who arrived at a weighted mean $[\mathrm{M} / \mathrm{H}]=-0.98 \pm 0.10$, for $T_{\text {eff }}=3570 \mathrm{~K}$, the latter derived from a recent angular diameter measurement by Ségransan et al. (2003). In a later paper, Woolf \& Wallerstein (2005) revised the metallicity upward slightly to $[\mathrm{M} / \mathrm{H}]=-0.86$, with $[\mathrm{Fe} / \mathrm{H}]=-0.99 \pm 0.04$ and $[\mathrm{Ti} / \mathrm{Fe}]=+0.18$. Using photometry from Leggett (1996), Dawson \& De Robertis (2004) estimated the bolometric flux to be $2.52 \times 10^{-11} \mathrm{~W} \mathrm{~m}^{-2}$; that, combined with an angular diameter of 0.696 mas obtained by application of the infrared flux method, led to $T_{\text {eff }}=3535 \mathrm{~K}$. (An advantage of this approach is that it obviates the need for limbdarkening corrections, some of which appear to be dubious, at least in giant stars, as demonstrated by the analysis by Fields et al. (2003) of the EROS BLG-2000-5 microlensing event.) The corresponding stellar radius is $0.293 R_{\odot}$, based on the large Hipparcos parallax of 255.26 \pm 0.86 mas (Perryman et al. 1997). The mass is found from the Delfosse et al. (2000) relation between mass and $K$-band luminosity to be $0.274 M_{\odot}$, so that $\log g=4.94$. Woolf and Wallerstein adopted very similar values of $T_{\text {eff }}$ and $\log g$; namely, $3570 \mathrm{~K}$ and 4.96 . Because it is based on reliable stellar parameters and the use of up-to-date materials and methods, the metal abundance found by Woolf \& Wallerstein (2005) is adopted here.

\section{The spectrum of Kapteyn's Star}

An optical spectrum of KS, covering the range $0.3425 \mu \mathrm{m} \leq$ $\lambda \leq 0.7430 \mu \mathrm{m}$, was taken at the Siding Spring observatory on October 30, 2002 by M.S. Bessell, using the $2.3 \mathrm{~m}$ Advanced Technology Telescope and the Dual-Beam Spectrograph (DBS). The DBS is described by Rodgers et al. (1988). Briefly, the visible band, from $3200-9000 \AA$ is divided by a dichroic beamsplitter which switches from transmission to

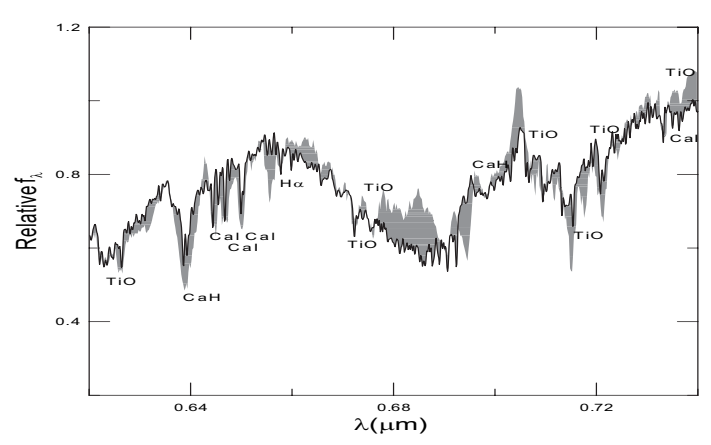

Fig. 1. A portion of the red spectrum of Kapteyn's Star(solid line). Certain conspicuous features have been labelled. The "free" boundary of the shaded region is defined by the synthetic spectrum described in the text; the shaded region shows the difference between the two. The spectra are scaled so that the total fluxes in each are the same over this wavelength interval.

reflection near a wavelength of $6000 \AA$; the beams feed similar spectrographs equipped with blue- and red-optimized detectors. The IR spectrum was taken on October 14, 2003 with the same telescope and the Cryogenic Array Spectrometer/Imager (CASPIR). The wavelength coverage in this case was between 1 and $2.5 \mu \mathrm{m}$. The $I J, J H$, and $H K$ cross-dispersed grisms were used, giving a spectral resolution of 1100 . The blue and red optical spectra were bias-subtracted and flat-field corrected and then divided by the spectrum of a hot star to remove to first order the response curve of the system. The data were then scaled so that the $B, V$, and $R$ apparent magnitudes computed from the spectrum were consistent with the observed magnitudes given by Leggett (1992), within a few hundredths of a magnitude. After the basic reductions, telluric features were removed from the IR spectra by division by the spectrum of HR 721 (B5IV); the hydrogen lines were first removed from the spectrum of the hot star after fitting their line shapes with Voigt profiles. The orders were then pieced together to produce a single spectrum which was flux-calibrated by multiplying by the continuum of a B5 IV star. The result was then scaled so that the computed $J$ magnitude agrees with that given by Leggett (1992).

In Fig. 1, part of the KS SED is shown. A number of atomic and molecular features are labelled. Most of the identifications are taken from Table 4 of Leggett et al. (1996) and the exact wavelengths can be found there. A few others are based on data from the U. S. National Institute of Standards and Technology (NIST) atomic spectra database ${ }^{1}$. Knowing the effective temperature, metal abundance and surface gravity of KS, we can compare its spectral energy distribution with a synthetic spectrum based on the new GAIA model atmosphere grid, which supersedes the NextGen grid. For simplicity, we adopted $\log g=5.00$ (the results are not sensitive to changes of one or two tenths of a dex), $[\mathrm{M} / \mathrm{H}]=-0.90$, and $T_{\text {eff }}=3550 \mathrm{~K}$, with $\alpha=+0.20$ (based on the $[\mathrm{Ti} / \mathrm{Fe}$ value cited above) and interpolated in the GAIA grid to arrive at a representative model spectrum. The result was then redshifted to account for the radial velocity of the star and smoothed using an

1 The URL is http://physics.nist.gov/PhysRefData/ contents.html 


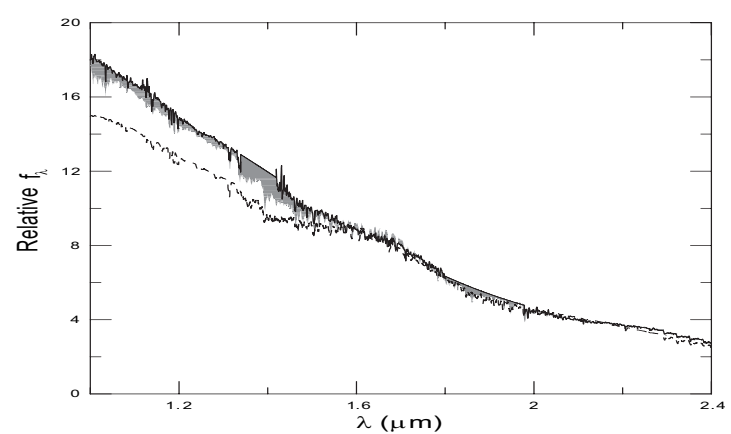

Fig. 2. The near-IR SED of Kapteyn's Star (solid line) compared with the synthetic spectrum described in the text. As in Fig. 1, the actual GAIA synthetic spectrum is not directly plotted. The dashed line represents a NextGen synthetic spectrum corresponding to $T_{\text {eff }}=3550 \mathrm{~K}$, and $[\mathrm{M} / \mathrm{H}]=-0.90$; the superiority of the new models is apparent. The SEDs have been adjusted so that they have the same flux density at $K$.

adjacent averaging scheme to approximate the resolution of the actual spectra. To avoid clutter in Fig. 1, the synthetic spectrum is not plotted directly, but it defines the "free" boundary of the shaded area, which shows the difference between it and the observed SED (solid line). The scaling is such that the total fluxes (over this wavelength interval) are equal. It is clear that the new GAIA models represent a very significant improvement over their NextGen predecessors, not only in the optical part of the spectrum, but also in the infrared. There, the NextGen models predicted significantly less flux in the $J$ - and $H$-bands than is actually observed; this was a consequence of missing opacity in the optical part of the spectrum, which allowed radiation to escape there, rather than at longer wavelengths.

The improvement due to the new models is illustrated in Fig. 2, which shows the stellar SED (solid line) from 1 to $2.4 \mu \mathrm{m}$. Two regions of extreme telluric absorption, extending from about 1.34 to $1.42 \mu \mathrm{m}$, and from 1.79 to $1.98 \mu \mathrm{m}$, have been excised. The stellar and model spectra were scaled so that the respective flux densities at $K(2.179 \mu \mathrm{m})$ are equal - see Berriman et al. (1992) for the rationale underlying this assumption. As in Fig. 1, the GAIA model spectrum is not displayed directly. We have, however, plotted as a dashed line a NextGen-based synthetic spectrum corresponding to $[\mathrm{M} / \mathrm{H}]=$ -0.9 and $T_{\text {eff }}=3550 \mathrm{~K}$; it has been scaled in the same way as the GAIA spectrum. The superiority of the latter over virtually the entire wavelength range depicted is clear, and the good agreement between theory and observation strengthens our confidence that the fundamental parameters of the star are correct.

Structure models of KS, on the other hand, do not agree very well with the observations. For example, interpolation in the grid published by Baraffe et al. (1997) yields (at age $10 \mathrm{Gyr}$ ) for a mass of $0.274 M_{\odot}$ with $[\mathrm{M} / \mathrm{H}]=-1.00$ an effective temperature of $3811 \mathrm{~K}$, with $M_{\mathrm{bol}}=+9.30$ and a radius of $0.270 R_{\odot}$ - too hot, too luminous (KS has $M_{\mathrm{bol}}=+9.52$ ) and too small. The persistent problem of theoretical underestimates of the radii of low-mass stars has been discussed by a number of authors, including Torres \& Ribas (2002), who remarked that the quality of recent observational data makes the shortcomings of the models more evident.
Table 1. General Galaxy model parameters.

\begin{tabular}{lrrrr}
\hline \hline & $M_{\mathrm{dh}}$ & $M_{\mathrm{n}}$ & $M_{\mathrm{b}}$ & $M_{\mathrm{oh}}$ \\
\hline Mass & 6550 & 400 & 300 & 30000 \\
$\quad$ Radial scale, kpc & $2.877^{a} / 6.0^{b}$ & 0.3 & 3.5 & 33.5 \\
\cline { 3 - 5 } & & & & \\
${ }^{a}$ Scale size of the disk. & & & & \\
${ }^{b}$ Scale size of inner halo. & & &
\end{tabular}

Table 2. Galaxy model disk parameters.

\begin{tabular}{|c|c|c|c|}
\hline Component & Thin disk & Normal disk & Thick disk \\
\hline Relative weight ${ }^{a}$ & 0.155 & 0.600 & 0.245 \\
\hline$z$-scale height ${ }^{b}$ & 0.125 & 0.325 & 1.000 \\
\hline
\end{tabular}

\section{The Galaxy model parameters}

The Galaxy potential presented here is of the same family used in Carlberg \& Innanen (1987) and in Caranicolas \& Innanen (1991). In those papers, it was shown that several nearby stars with retrograde Galactic orbits are very close to the chaotic threshold. One of these is KS. The primary cause of the chaos is the supposed concentration of mass $\left(\approx 10^{10} M_{\odot}\right)$ near the Galactic centre. Thus a galaxy model was constructed by one of us (KI) based on recent estimates of the relevant parameters, and which incorporates certain resonances (described below) that should test the nature of the chaotic threshold. With such resonances in the model, it is then possible to introduce periodic perturbations so as to induce the possibility of chaos, in the usual way. Unless otherwise noted, lengths are expressed in $\mathrm{kpc}$, velocities in $\mathrm{km} \mathrm{s}^{-1}$, masses in units of $2.316 \times 10^{7} M_{\odot}$ and densities in $M_{\odot} \mathrm{pc}^{-3}$. The basic adopted parameters of the model are: $R_{\odot}=8.0, V_{\odot}=220$, and $\rho_{\odot}=0.118$. The last number is taken from the analysis by Holmberg \& Flynn (2000). The first two values retain their well-known uncertainties, and can readily be varied to maintain the resonances described below. If, in addition, $\Omega_{\odot}, \kappa_{0}, \Omega_{z}$ are the respective angular frequencies of the local standard of rest, the classical Galactic local epicycle and the vertical oscillation, then our adopted model is designed to produce the resonances $\kappa_{0}: \Omega_{\odot}=3: 2$, and $\Omega_{z}: \kappa_{0}=2: 1$. In order to produce these resonances in the model, the rotation curve at $R_{\odot}$ must rise slowly at the rate of $3.5 \mathrm{~km} \mathrm{~s}^{-1} \mathrm{kpc}^{-1}$. The resulting Oort rotation constants at $R_{\odot}$ are $(\mathrm{A}, \mathrm{B})=(12.0,-15.5) \mathrm{km} \mathrm{s}^{-1} \mathrm{kpc}^{-1}$. The possibility of a locally rising rotation curve has been noted among others by Caldwell \& Ostriker (1981), although in an unrelated context. With these choices, a non-linear, partly trial-and-error process is used to construct the model galaxy. The results are summarised in Tables 1 and 2. The subscripts "dh, n, b, oh" refer to the composite disk-halo, nucleus, bulge, outer and inner halo. The inner halo is part of the composite disk halo. All of these, except the disk, are taken to be spherical. The model rotation curve is shown in Fig. 3. Co-rotation occurs near $R=3.23 \mathrm{kpc}$.

Next, we introduce a purely empirical bar/axisymmetric distortion (BAD), rotating in the inner Galaxy at a fixed 


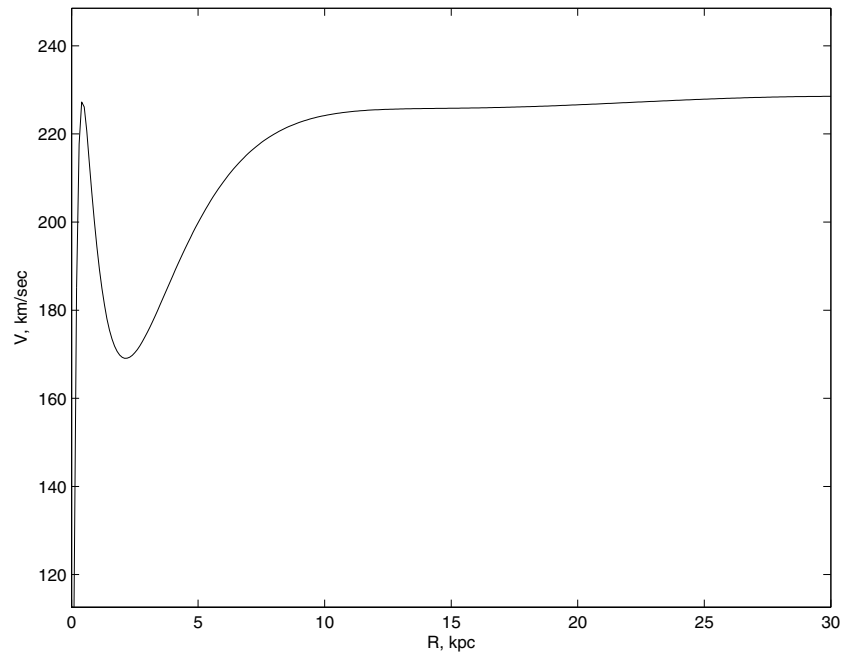

Fig. 3. The rotation curve of the model galaxy.

angular rate $\Omega_{b}$ of $1 / 2$ of the angular speed of the local standard of rest $\Omega_{\odot}$, i.e. $\Omega_{b}=\Omega_{\odot} / 2$. Some sort of BAD is now generally believed to exist in the inner Galaxy and this choice will ensure that some pumping of resonances can take place. The amplitude of this empirical BAD can be taken to be less than a few percent of the usual axisymmetric velocity components. Although this is clearly not self-consistent, it should provide a first approximation to any chaotic activity during 1, 2 or 3 Galactic years near the Sun. This choice of the period of the $\mathrm{BAD}$, together with the other resonances, makes the index $m= \pm 3 / 2$ (following the nomenclature of Binney $\&$ Tremaine 1987). It also has the interesting effect of moving the inner Lindblad Resonance (ILR) to the position $R_{\odot}$. The value $m=3 / 2$ indicates that the BAD is not the "standard" value $m=2$ expected for a simple bar. This BAD will also affect all of the local stars (i.e. the local standard of rest, LSR) in the same way. A small, periodic oscillation of the LSR, mainly radial, results. This situation is not new in the study of the dynamics of the Galaxy. An outward movement of the LSR of about $7 \mathrm{~km} \mathrm{~s}^{-1}$ was proposed by Kerr (1963) to explain the observed asymmetry in the $21 \mathrm{~cm}$ rotation curves derived from the northern and southern neutral hydrogen surveys. That asymmetry still exists. Kerr proposed radial outward motion to reconcile the asymmetry. This sort of oscillation is not part of classical Galactic dynamics, and its implications, as well as the implications of the ILR location at $R_{\odot}$, are beyond the scope of this paper. The above choices of the resonant BAD make our galaxy model non-standard, but not truly radical. The analysis will provide results with the BAD turned both on and off, for comparison. The extent of chaos will be measured in the usual way, using the orbit's surface-of-section.

\section{The Galactic orbit of Kapteyn's star}

The starting conditions for all of the computed orbits assume the standard velocity components of the solar motion to be 10.0 toward the Galactic centre, 7.2 in the direction of Galactic rotation and 5.2 toward the north Galactic pole, all in $\mathrm{km} \mathrm{s}^{-1}$. With the Hipparcos astrometry (Perryman et al. 1997)

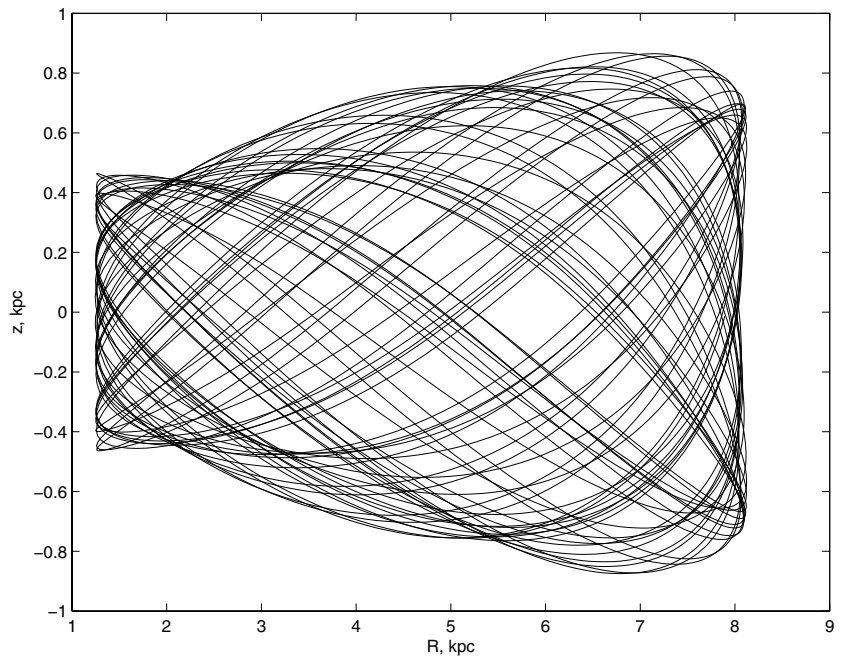

Fig. 4. The KS orbit in the meridional coordinate system with no perturbation.

and the radial velocity of $245.2 \pm 0.4 \mathrm{~km} \mathrm{~s}^{-1}$ (Nidever et al. $2002)$, the components $(U, V, W)$ of the space velocity of $\mathrm{KS}$ are $(21.1 \pm 0.3,-287.8 \pm 0.3,-52.6 \pm 0.3) \mathrm{km} \mathrm{s}^{-1}$ along the same axes.

Figure 4 illustrates the motion of KS in the rotating (meridional) coordinate system of our Galaxy model, with no perturbation. This orbit is mainly one with a quasi-third integral producing the usual near box-like configuration. Figure 5 is the Poincaré surface of the section corresponding to Fig. 4. The onset of chaos is evident. Figure 6 illustrates the same motion in the same model, but with an empirical, periodic perturbation applied to its motion, through the action of the BAD described above, with an amplitude of $1 \%$ of the non-perturbed $z$-velocity. The chaotic behaviour is now readily apparent. Figure 7 is the Poincare surface of the section corresponding to the orbit of Fig. 6. In Fig. 8, the effect of a $0.5 \%$ perturbation to the radial velocity $\mathrm{d} R / \mathrm{d} t$ of $\mathrm{KS}$ is shown. The results illustrate that radial motions in the Galaxy are, in fact, somewhat more sensitive to perturbations than are vertical motions. Figure 9 shows the corresponding surface of the section. The chaotic behavior is evident.

\subsection{The origin of Kapteyn's star}

Since the kinematics and the dynamics of KS stand out among those of its neighbours, it is natural to look for the underlying causes; some work along these lines has already been done, e.g., Carlberg \& Innanen (1987); Caranicolas \& Innanen (1991). The orbit of KS is unique among the Sun's nearest neighbours; clearly, the star formed elsewhere in the Galaxy, and happens now to be in transit through the solar neighbourhood. There have been many studies of the dynamics, orbits and metallicties of globular clusters (e.g., Tsuchiya et al. 2003; Dinescu et al. 1999; Odenkirchen et al. 1997). We searched for the origin of KS by seeking a globular cluster whose metal abundance and kinematics correspond to those of the star. To do this, the most recently updated version of the catalogue 


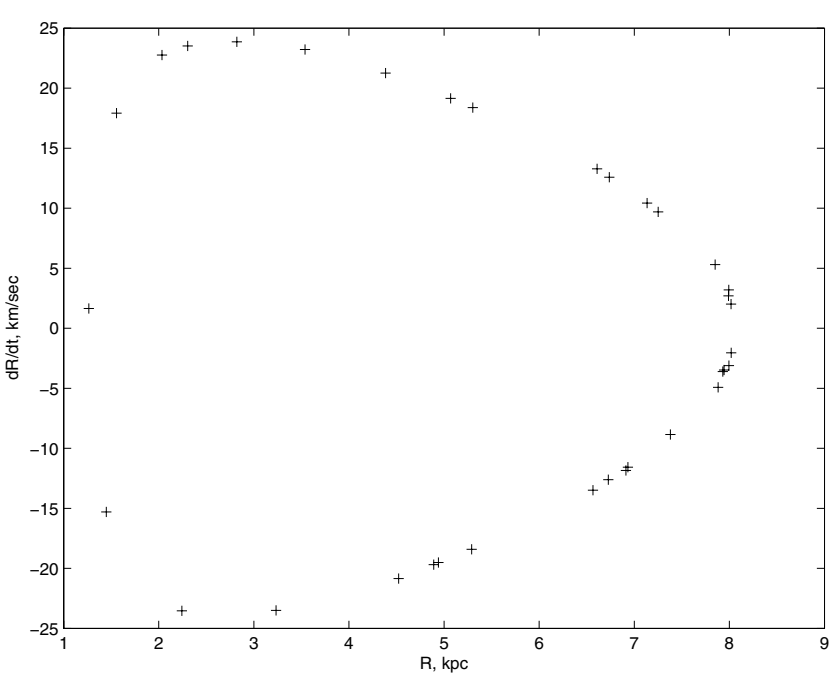

Fig. 5. The Poincaré surface of the section of the orbit in Fig. 4.

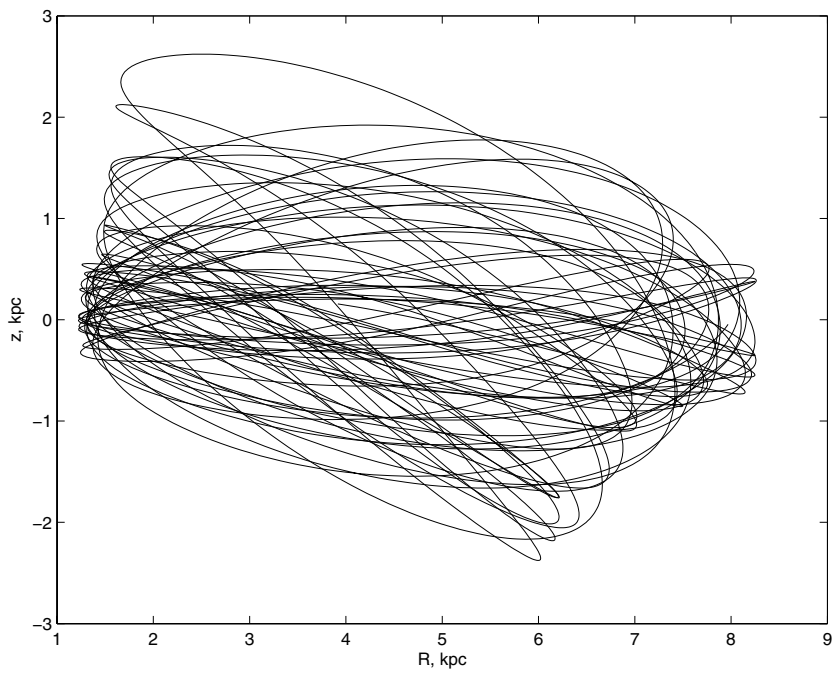

Fig. 6. Same orbit as Fig. 4, but with a $1 \%$ perturbation applied to the non-perturbed $z$-velocity.

of globular clusters by Harris (1996) was used ${ }^{2}$. For a first pass, we set metallicity limits of $-1.50<[\mathrm{M} / \mathrm{H}]<-0.50$, narrowing the sample to 72 clusters out of 150 , From among these, we identified 38 within $8.5 \mathrm{kpc}$ of the Galactic centre, selecting, where possible, those that move on retrograde orbits. However, it soon became clear that the most plausible source of KS is $\omega$ Cen (NGC 5139), even though it failed initially to qualify in terms of metallicity. This giant cluster is anomalous in many ways, especially in its chemical inhomegeneity; Norris et al. (1996) showed that its metallicity distribution is at least bimodal, with one peak at $[\mathrm{Fe} / \mathrm{H}] \approx-1.60$, and a smaller peak at $[\mathrm{Fe} / \mathrm{H}] \approx-1.20$. Moreover, there is a long tail that extends to $[\mathrm{Fe} / \mathrm{H}] \approx-0.5$, a range that comfortably includes the metallicity of KS. Tsuchiya et al. (2003) explored an accretion model for the origin of $\omega$ Cen, simulating the evolution of a dwarf galaxy captured by the Milky Way. They showed that a dwarf satellite whose initial mass was $8 \times 10^{9} M_{\odot}$, with

\footnotetext{
2 The URL is
}

http://www . physics.mcmaster.ca/Globular.html

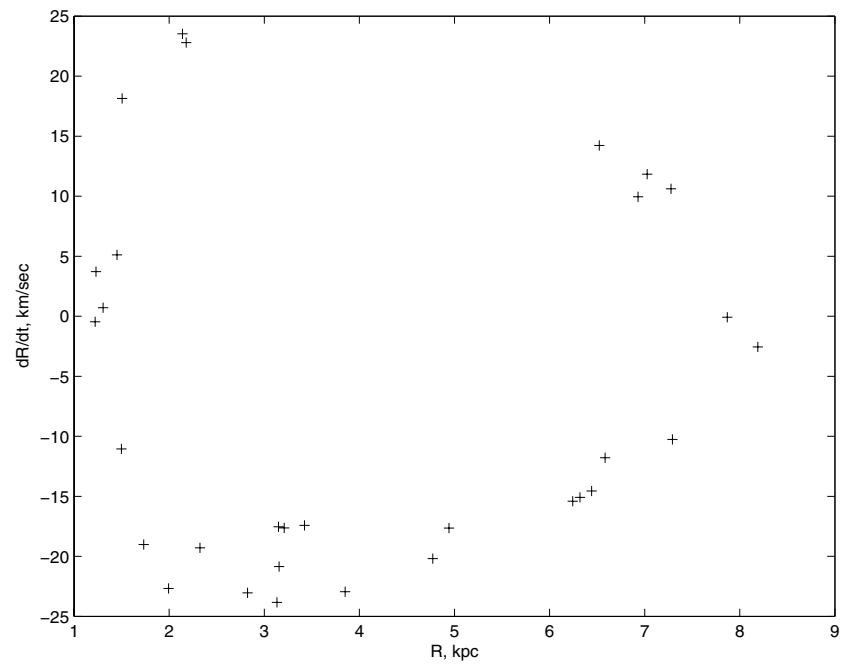

Fig. 7. The Poincaré surface of the section of the orbit in Fig. 6.

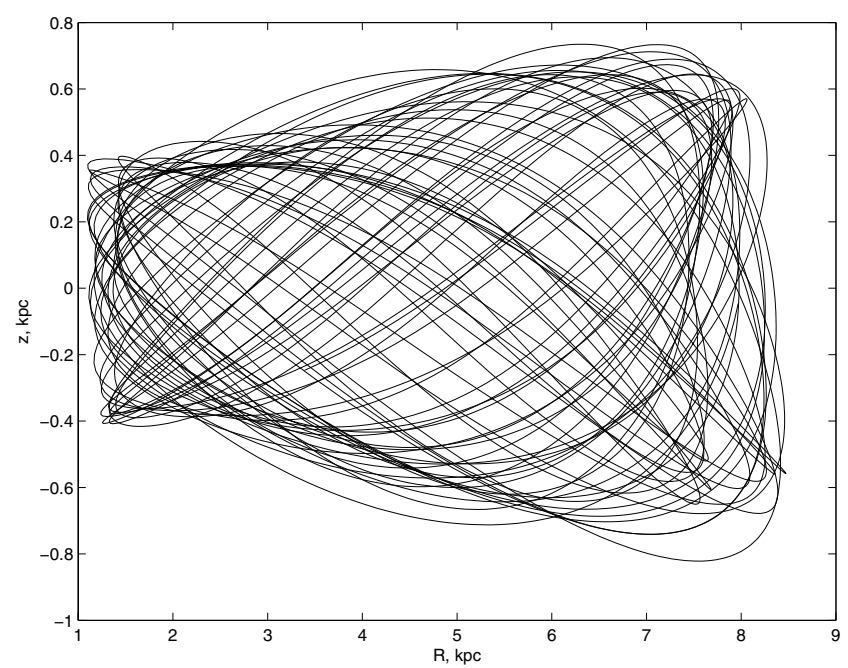

Fig. 8. Same orbit as Fig. 4, but with a $0.50 \%$ perturbation applied to the non-perturbed $R$-velocity.

a Hernquist density profile of $r_{1 / 2}=1.414 \mathrm{kpc}$, and a nuclear mass of $10^{7} M_{\odot}$ can be dragged into an orbit very like that of $\omega \mathrm{Cen}$; what remains is a central nucleus whose mass is of the same order of magnitude as that of the cluster. In this scenario, KS would be among the stars stripped from the dwarf satellite during its approach to its final orbit. Although it would be very difficult to prove conclusively that $\omega$ Cen is the birthplace of KS, we suggest that it is a reasonable possibility, and that it is not surprising that we should see one of its primordial members near the Sun at this epoch. There is one other, rather primitive, statistical point that can be made: if KS-like stars are uniformly distributed throughout the inner, spherical $10 \mathrm{kpc}$ volume of the Galaxy, with a space density $\sim 1$ in each volume of radius $10 \mathrm{pc}$, then there should be about a million such stars in the larger volume of space. That constitutes the mass of one large globular cluster, but it will not contribute significantly to the total mass of the Galaxy. 


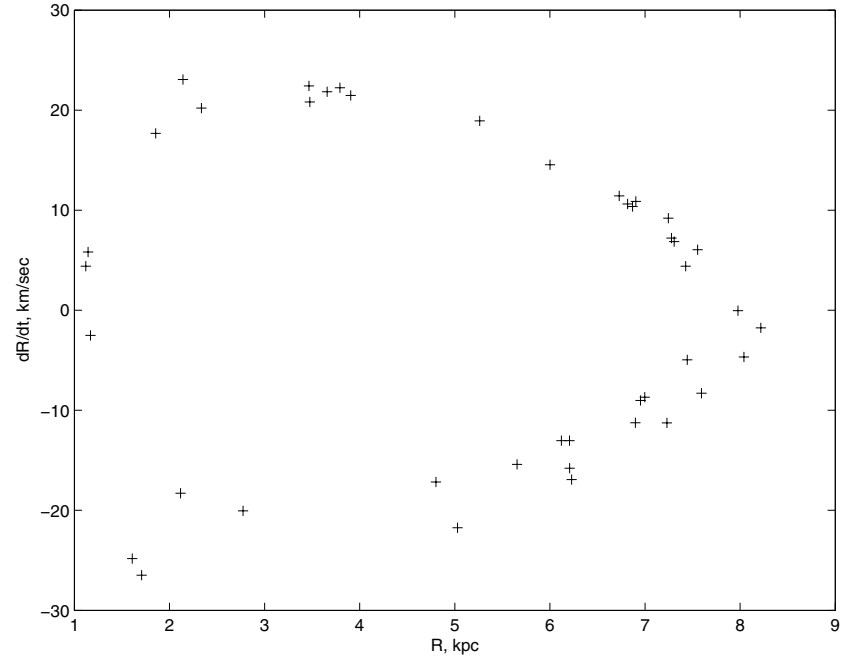

Fig. 9. The Poincaré surface of the section of the orbit in Fig. 8.

\section{Discussion and conclusions}

The basic properties of KS, i.e., its mass, its radius, and its metallicity seem now to be well established. Woolf \& Wallerstein (2004) showed that it is possible to locate regions of the SED that are largely or entirely free from molecular absorption, and used the equivalent widths of atomic lines therein to arrive at a weighted mean $[\mathrm{M} / \mathrm{H}]=-0.86$ (they do not give an uncertainty, but something close to 0.10 dex seems likely). We have compared our low-resolution spectra, both optical and infrared, with synthetic spectra based on the parameters cited in the text. The new GAIA models represent the observations rather well, and are a very significant improvement over the NextGen models, now obsolete, which preceded them. Even the most sophisticated structure models, however, underestimate the stellar radius, and overestimate its effective temperature.

Using its accurately-known space velocity, we have analyzed the Galactic orbit of KS. We speculate that the star may have formed in a dwarf satellite of the Milky Way, which was then dragged into a low-energy orbit, losing most of its mass in the process. We suggest that the remnant may be the globular cluster $\omega$ Cen, whose retrograde orbit is similar to that of KS, and which includes among its established members stars with the metallicity of KS.

Acknowledgements. This work has been supported by the Yrjö, Vilho ja Kalle Väisälä Foundation (EK), and by grants (to KAI, MMDR and PCD) from the Natural Sciences and Engineering Research Council (NSERC) of Canada. We thank Dr. Mike Bessell for help with the observations taken at SSO. K.A.I. is very grateful to Dr. Kris Innanen for a great deal of assistance in revising and updating various computer programs to greatly facilitate sections of the paper. KAI is also grateful to Dr. Seppo Mikkola for the hospitality that he enjoyed at the Tuorla Observatory in the spring of 2003. Finally, we are indebted to Dr. Peter Hauschildt for making available to us new synthetic spectra in advance of publication, and to an anonymous referee whose comments have strengthened this paper significantly.

\section{References}

Adams, W. S., \& Joy, A. H. 1924, PASP, 36, 141

Baraffe, I., Chabrier, G., Allard, F., \& Hauschildt, P. 1997, A\&A, 327, 1054

Berriman, G., Reid, N., \& Leggett, S. K. 1992, ApJ, 392, L32

Binney, J., \& Tremaine, S. 1987, Galactic Dynamics (Princeton: Princeton University)

Caldwell, J. A. R., \& Ostriker, J. P. 1981, ApJ, 251, 61

Caranicolas, N., \& Innanen, K. A. 1991, AJ, 102, 1343

Carlberg, R. G., \& Innanen, K. A. 1987, AJ, 84, 666

Curtis, H. D. 1909, Lick Obs. Bull., 5, 133

Dawson, P., \& De Robertis, M. 2004, AJ, 127, 2909

Delfosse, X., Forveille, T., Ségransan, D., et al. 2000, A\&A, 364, 217

Dinescu, D. I., Girard, T. M., \& van Altena, W. F. 1999, AJ, 117, 1792

Eggen, O. J. 1977, ApJ, 215, 812

Eggen, O. J. 1978, ApJ, 221, 881

Eggen, O. J. 1997, AJ, 114, 825

Fields, D. L., Albrow, M. D., An, J., et al. 2003, ApJ, 596, 1305

Gizis, J. E. 1997, AJ, 113, 806

Harris, W. E. 1996, AJ, 112, 1487

Hauschildt, P. H., Allard, F., \& Baron, E. 1999, ApJ, 512, 377

Holmberg, J., \& Flynn, C. 2000, MNRAS, 313, 209

Jones, H., Pavlenko, Y., Viti, S., \& Tennyson, J. 2002, MNRAS, 330, 675

Kapteyn, J. C. 1897, Astr. Nach. No. 3466, 145, 159

Krawchuk, C. A. P., Dawson, P. C., \& De Robertis, M. M. 2000, AJ, 119,1956

Kerr, F. J. 1963, MNRAS, 123, 327

Kuiper, G. P. 1940, ApJ, 91, 269

Leggett, S. K. 1992, ApJS, 82, 351

Leggett, S. K., Allard, F., Berriman, G., Dahn, C. C., \& Hauschildt, P. H. 1996, ApJS, 104, 117

Nidever, D. L., Marcy, G. W., Butler, R. P., Fischer, D. A., \& Vogt, S. S. 2002, ApJS, 141, 503

Mould, J. R. 1976, ApJ, 210, 402

Norris, J. E., Freeman, K. C., \& Mighell, K. L. 1996, ApJ, 487, L187

Odenkirchen, M., Brosche, P., Geffert, M., \& Tucholke, H.-J. 1997, New Astron., 2, $477 \mathrm{O}$

Partridge, H., \& Schwenke, D. W. 1997, J. Chem. Phys., 106, 4618

Perryman, M. A. C., Lindegren, L., Kovalevsky, J., et al. 1997, A\&A, 323, L49

Proust, D., \& Foy, R. 1988, Ap\&SS, 145, 61

Rodgers, A. W., Conroy, P., \& Bloxham, G. 1988, PASP, 100, 626

Ségransan, D., Kervella, P., Forveille, T., \& Queloz, D. 2003, A\&A, 397, L5

Torres, G., \& Ribas, I. 2002, ApJ, 567, 1140

Tsuchiya, T., Dinescu, D. I., \& Korchagin, V. 2003, ApJ, 589, L29

Wing, R. F., Dean, C. A., \& McConnell, D. J. 1976, AJ, 205, 186

Woolf, V. M., \& Wallerstein, G. 2004, MNRAS, 350, 575

Woolf, V. M., \& Wallerstein, G. 2005, MNRAS, 356, 963 